Meta

Journal des traducteurs

Translators' Journal

\title{
Langue, immigration, culture : paroles de la banlieue française
}

\section{Nadia Duchêne}

Volume 47, numéro 1, mars 2002

URI : https://id.erudit.org/iderudit/007989ar

DOI : https://doi.org/10.7202/007989ar

Aller au sommaire du numéro

\section{Éditeur(s)}

Les Presses de l'Université de Montréal

ISSN

0026-0452 (imprimé)

1492-1421 (numérique)

Découvrir la revue

Citer cet article

Duchêne, N. (2002). Langue, immigration, culture : paroles de la banlieue française. Meta, 47(1), 30-37. https://doi.org/10.7202/007989ar

\section{Résumé de l'article}

L'étude du langage envisagé comme système de signes constitue le champ par excellence de la linguistique. Le parler des banlieues, en tant que variante du français utilisée dans un milieu donné, certes, n’est pas la seule nouveauté de la langue française actuelle et semble être l'objet d'un intérêt grandissant parmi les linguistes. Nous nous proposons dans notre article d'aborder ce phénomène de langue au centre de problématiques peut-être davantage sociales et culturelles que linguistiques. Lorsque nous aurons délimité les contours de cette variation langagière dans le cadre de la langue française et de sa communauté, nous étudierons un corpus et analyserons entre autres la fonction cryptique, le pouvoir symbolique et les figures de rhétorique qui caractérisent ce parler ; ceci depuis la perspective de la création et de l'affirmation d'une identité immergée dans une situation sociale bien spécifique. 


\title{
Langue, immigration, culture: paroles de la banlieue française
}

\author{
NADIA DUCHÊNE \\ Université de Huelva, Huelva, Espagne
}

\begin{abstract}
RÉSUMÉ
L'étude du langage envisagé comme système de signes constitue le champ par excellence de la linguistique. Le parler des banlieues, en tant que variante du français utilisée dans un milieu donné, certes, n'est pas la seule nouveauté de la langue française actuelle et semble être l'objet d'un intérêt grandissant parmi les linguistes.

Nous nous proposons dans notre article d'aborder ce phénomène de langue au centre de problématiques peut-être davantage sociales et culturelles que linguistiques. Lorsque nous aurons délimité les contours de cette variation langagière dans le cadre de la langue française et de sa communauté, nous étudierons un corpus et analyserons entre autres la fonction cryptique, le pouvoir symbolique et les figures de rhétorique qui caractérisent ce parler; ceci depuis la perspective de la création et de l'affirmation d'une identité immergée dans une situation sociale bien spécifique.
\end{abstract}

\begin{abstract}
The study of language, considered as a system of signs, belongs above all to the field of linguistics. This "suburban" way of speaking, as a variant, is certainly not the only new form as far as the French language is concerned, although linguists appear to be increasingly interested in it. In this paper, we intend to tackle this language phenomenon which is perhaps centred on questions more social and cultural than linguistic. Once we have framed the outlines of this language variation within the field of the French language and its community, we will study a corpus and we will analyze the cryptic function, the symbolic power and the figures of speech which characterize this way of speaking. We will do it both from the perspective of creation and from the affirmation of an identity within a very specific social situation.
\end{abstract}

\section{MOTS-CLÉS/KEYWORDS}

banlieue, figures de rhétorique, fonction cryptique, pouvoir symbolique, variation langagière

L'étude du langage envisagé comme système de signes relève par excellence du champ de la linguistique. Le parler des banlieues, en tant que variante du français, ne constitue, certes, pas la seule nouveauté concernant notre langue mais se trouve à l'origine d'un intérêt grandissant chez les linguistes.

Nous nous proposons dans notre article d'aborder ce phénomène de langue au centre de problématiques peut-être davantage sociales et culturelles que linguistiques. Lorsque nous aurons délimité les contours de cette variation langagière dans le cadre de la langue française et de sa communauté, nous étudierons un corpus et analyserons entre autres, la fonction cryptique, le pouvoir symbolique et les figures de rhétorique qui caractérisent ce parler; ceci depuis la perspective de la création et de l'affirmation d'une identité immergée dans une situation sociale bien spécifique. 
Dans la mesure où notre intérêt porte sur le langage des banlieues, il convient tout d'abord d'aborder deux concepts clés, à savoir «l'usage de la langue» ou encore les variétés de cette dernière pour ensuite définir ce que l'on entend par «communauté linguistique».

La variété n'est, en effet, pas inconciliable avec les notions de langue et de communauté. Elle peut être liée à divers facteurs extralinguistiques dont l'importance varie. La variété peut être d'ordre régional, social, stylistique, temporel... Pour notre part, nous nous intéresserons plus particulièrement à la variété de type social. Dans toutes les sociétés, les individus diffèrent les uns des autres par leurs pratiques langagières. Certaines de ces différences sont idiosyncratiques mais d'autres sont systématiquement associées à des groupes de locuteurs particuliers. Les différences les plus évidentes résident dans la distinction de sexe et de niveau de développement: les femmes ne s'expriment pas de la même façon que les hommes tout comme les enfants ne parlent pas comme les adultes. Il s'agit dans ce cas de différences essentiellement biologiques mais dans la plupart des sociétés, les variations langagières vont au delà de ces dimensions et relèvent de conventions et de symboles sociaux. Ainsi les aspects sociosymboliques du langage quant à son usage ont une fonction emblématique de sorte qu'ils identifient le locuteur à un groupe particulier ou lui accordent une identité sociale. Au sein de la société certaines de ces divisions sociolinguistiques sont directement mises en relation avec les différences de prestige social et de pouvoir. Citons à ce propos Pierre Bourdieu:

On doit se garder d'oublier que les rapports de communication par excellence que sont les échanges linguistiques sont aussi des rapports de pouvoir symbolique où s'actualisent les rapports de force entre les locuteurs ou leurs groupes respectifs (1982: 14).

La langue dans ses usages révèle des variations; c'est ainsi que le locuteur effectue un choix parmi les variétés, les codes de la langue, notamment en fonction de son statut social, de la situation d'échange plus ou moins formelle et du style qu'il souhaite adopter. À ce titre la définition de «variété» proposée par Ferguson (1971) nous semble assez précise. Selon ce linguiste, la variété est un ensemble de patrons linguistiques suffisamment homogènes de sorte à être analysés au moyen de techniques linguistiques de type synchronique. Cela dit, les types de variétés sont relativement hétérogènes puisque la sociolinguistique étudie principalement les langues, les dialectes, les parlers, les sociolectes, les styles ou registres.

Lorsque nous parlons des facteurs à l'origine des variantes linguistiques, nous faisons également référence aux phénomènes propres à une communauté linguistique. Le terme "communauté» nous rapporte immédiatement au fait de partager. Ainsi une communauté linguistique est composée d'un ensemble de locuteurs qui partagent en effet, non seulement une langue, mais aussi un ensemble de normes et de valeurs d'ordre sociolinguistique. Ils adoptent des attitudes linguistiques semblables, des règles d'usage communes. Les membres de cette communauté sont donc capables de se reconnaître à travers leurs pratiques langagières. Selon la sociolinguistique actuelle, une communauté linguistique est principalement une communauté au sein de laquelle il existe un consensus, une sorte de syntonie entre individus différents et où le conflit est minimisé. Christian Baylon parle de «normes partagées »:

Le critère de normes partagées fait référence à un ensemble commun de jugements évaluatifs, une connaissance à l'échelle de la communauté de ce qui est considéré 
comme bon ou mauvais et ce qui est approprié à tel type de situation socialement définie. Ces types de normes peuvent exister pour tous les aspects de comportement social, mais ce qui nous intéresse ici ce sont les normes linguistiques (1996: 91).

Après avoir dégagé les traits principaux des concepts de variété et de communauté linguistiques et avant d'envisager le langage de la banlieue dans sa structure et son fonctionnement, il convient de s'interroger sur le phénomène de la banlieue au sein du tissu social français. Citons pour ouvrir le sujet, les propos de Hervé Vieillard-Baron :

Pour mémoire, la banlieue, un peu comme une série dramatique, fut d'abord un chantier commencé aux portes de la ville, mais jamais terminé; ensuite une histoire plutôt pauvre sans origine clairement attestée, sans intrigue bien définie ni dénouement prévisible; enfin, un rassemblement d'acteurs: décideurs politiques, peuple «citoyen", minorités ethniques et ombres clandestines (1996: 9).

La banlieue évoque ainsi en France un produit social composé de communes périphériques qui renvoient tout d'abord au discrédit, à la fracture sociale, aux tensions raciales et donc à la crise économique. Depuis les années quatre-vingt, la banlieue est systématiquement associée à la marginalité et à l'exclusion. Elle s'érige en symbole de mal social, territoire de transition entre la ville et la campagne, elle est aujourd'hui un espace varié dans lequel les nationalités et par conséquent les ethnies y sont très diversifiées. Des identités multiples s'y construisent et cherchent à s'exprimer dans la société française. Les groupes sociaux qui habitent la banlieue se situent au carrefour d'une identité réelle et à la fois symbolique. Malgré le fait qu'ils aient perdu de nombreuses références à leur pays d'origine, il n'en demeure pas moins qu'ils ne se considèrent pas tout à fait français. Selon Emmanuel Vaillant:

Quant à leurs enfants, français pour la plupart, ils se définissent souvent comme étant «entre deux cultures». L'une, réelle et quotidienne est le résultat de leur participation à la société dont ils sont membres. L'autre, plus diffuse, exprime une sorte de fidélité à des origines familiales, connues ou inconnues, mais bien présentes dans les esprits par la façon de se désigner ou d'être désignés: «beurs», «blacks», etc. (1996: 54).

Le jeune enfant d'immigré est donc confronté à une situation pernicieuse puisqu'il se trouve au centre de discours contradictoires. D'un côté, on souhaite l'intégrer en minimisant ce qu'on dit être sa culture, en méconnaissant une partie de sa personnalité; de l'autre, on lui demande de réussir à l'école en l'engageant à prendre garde à la culture qu'on lui inculque afin de rester fidèle à ses origines. C'est pourquoi il s'agit pour lui de gérer une identité aux facettes multiples. La banlieue, lieu de résidence de la plupart des populations immigrées en France, a développé une nouvelle langue que les linguistes se sont accordés à dénommer «langue de la banlieue » ou «langue contemporaine des cités ». Ce phénomène linguistique en tant qu'objet d'étude, date environ des années quatre-vingt-dix selon Henry Boyer (1997:22), spécialiste de ce sujet. À partir de cette époque, on verra se multiplier les dictionnaires, les articles de journaux et de revues spécialisées traitant cette situation sociolinguistique. Écoutons les propos de Pierre Merle dans son ouvrage Le prêt à parler:

Aujourd'hui? Pas un article de journal sur un sujet un peu pointu qui ne soit assorti de son indispensable glossaire. Pas une ethnie, pas une communauté, pas une banlieue qui échappe à la sagacité des faiseurs de lexiques derrière lesquels courent, voraces, et pour d'évidentes raisons commerciales, les dictionnaires classiques. La mode est au langage 
et le langage est à la mode. Notre siècle finissant n'a-t-il pas été, de la TSF à Internet, via bien entendu l'omniprésente télévision, celui de la communication? (1999:9)

Il y a donc bien une fascination pour le langage de la banlieue, comparable à celle qui exista à la fin du $\mathrm{XIX}^{\mathrm{e}}$ ou au début du $\mathrm{xx}^{\mathrm{e}}$ siècle pour l'argot. Afin de mieux en cerner la structure et le fonctionnement, il nous a semblé intéressant de recourir au «rap», devenu l'une des expressions les plus significatives pour l'objet de notre étude. Bien entendu, ce style de musique aux paroles hachées ne nous intéresse pas pour son rythme mais plutôt pour la composition de ses textes. Il s'agit bien, en effet, d'une véritable alchimie de mots évoquant une réalité multicolore, des scènes de vie urbaine, nous permettant de mieux connaître la vie dans les cités. Il convient de préciser à cet endroit que le rap s’inscrit dans le mouvement «Hip-Hop » venu des États-Unis, lequel regroupe à la fois la musique, la danse, les tags, etc. Il est devenu aujourd'hui un véritable mode de vie au sein des banlieues. À titre d'exemple, nous avons choisi un passage de la chanson intitulée Un été à la cité du groupe Ministère Amer :

Cette nuit, $j$ 'ai fait un rêve, à vrai dire un cauchemar,

Il faut que j'écrive ça fera sûrement un rap pour plus tard.

Il est midi, la chaleur fait monter chez moi l'odeur du cheb et cantonnais du deuxième, le couscous et colombo du troisième mélangés au saka du quatrième.

Comme le dit Jacques Chichi décontracte, à chaque étage, [ça sent la bouffe, une vie de ouf.

Dans mes escaliers, tout le monde a signé, d'autres ont pissé, [des chiens ont chié.

Il n'y a plus de respect donc la gardienne gueule, sa mère fait [des simagrées.

Ma famille crie (Trouve un métier).

Je dois m'évader.

Il est 13 heures je descends faire du biz dans le quartier (1997: 32).

Nous voyons que le langage est cru, les paroles ne sont assujetties à aucune norme académique, les rimes sont approximatives ainsi que la ponctuation. Le malaise de la vie dans la cité est exprimé sans pudeur aucune. Certains termes ou expressions appartiennent de toute évidence au registre familier ou vulgaire. Les thèmes les plus fréquents évoqués dans le rap sont la drogue, le chômage, la précarité, le racisme, le sexe, la violence, etc.; autant de termes qui définissent la banlieue.

Par ailleurs au gré de conversations tenues dans le métro parisien nous avons pu y découvrir une quantité non négligeable de mots «complexes» pour l'oreille d'un non-initié... La créativité n'a plus de limites. Citons à cet égard quelques termes pris au hasard:

Crouille: terme signifiant «arabe», qui provient d'une déformation de «rouilla », c'està-dire frère en arabe. Les Arabes s'appellent «frère» entre eux.

Greune: verlan de «nègre», «noir».

Hétiste: «désœuvré», "au chômage», du mot arabe «het» qui signifie littéralement «tenir le mur», en d'autres termes: «ne rien faire».

Quagibra: du verlan de «braquage».

Quepa: du verlan de «paquet» dans lequel est vendu la drogue.

Rose marron: se rapporte à l'héroïne.

Serrefesses: "CRS»; il s'agit probablement d'une déformation phonétique. 
TATI: sigle employé pour «Tout Arabe Transite Ici».

Guèze/Guèzeman: pure invention signifiant «maigre».

Ces termes ne constituent certes qu'un petit échantillon et la liste pourrait s'étendre à l'infini... Prétendre élaborer un dictionnaire à peu près complet de la langue des banlieues relève de la simple utopie compte tenu de la rapidité avec laquelle évolue ce langage. Des mots entendus aujourd'hui tomberont en désuétude demain et de nouvelles créations verront le jour. Cela dit, nous avons eu recours au dictionnaire Tchatche de banlieue (1998) élaboré par Philippe Pierre Adolphe, Max Mamoud et Georges Olivier Tzanos; ouvrage d'un grand intérêt pour les non-initiés... Sur un corpus de 729 mots, nous nous sommes consacrés dans un premier temps à l'analyse de l'origine des mots de la banlieue et nous avons obtenu les résultats présentés dans le tableau qui suit:

\begin{tabular}{|c|c|c|}
\hline ORIGINE & PROPORTION EN \% & EXEMPLES \\
\hline Africain & $0,5 \%$ & Bab; babtou (un blanc) / Larlarato (bête) \\
\hline Argot & $1,5 \%$ & $\begin{array}{l}\text { Calter (s'enfuir) / Radasse (fille ou femme; péj.) / } \\
\text { Trimard (travailleur) }\end{array}$ \\
\hline Veul & $1,5 \%$ & $\begin{array}{l}\text { Asmeuk (comme ça) / Enjs (les gens) / Atec } \\
\text { (écouter) }\end{array}$ \\
\hline Anglais américain & $2,5 \%$ & $\begin{array}{l}\text { Etre on fire (être en forme) / Move (branché) / } \\
\text { Sister (sœur) }\end{array}$ \\
\hline Arabe & $3,5 \%$ & Clebs (chien) / Flouze (argent) / Miskin (faible) \\
\hline Gitan ou tzigane & $3,5 \%$ & Pélo (pénis) / Caballo (étranger) / Moulo (gamin) \\
\hline Verlan & $18 \%$ & $\begin{array}{l}\text { Gueudin (dingue) / Quécro (croquer) / Chanmé } \\
\text { (méchant) }\end{array}$ \\
\hline Création & $69 \%$ & $\begin{array}{l}\text { Chaquin (travail) / Base (cité) / Pelouse (herbe - } \\
\text { cannabis) }\end{array}$ \\
\hline
\end{tabular}

* Les pourcentages ont été arrondis au centième supérieur.

L'origine de ces mots ne doit en rien nous surprendre étant donné la réalité de la présence étrangère en France. Pour ce qui est des procédés sémantiques, outre les emprunts cités ci-dessus, nous pouvons souligner le verlan (inversion de «à l'envers») qui consiste à inverser les syllabes d'un mot, sorte de codage linguistique; puis, le veul, autre procédé argotique qui verlanise de nouveau un mot déjà verlanisé. Le but est de rendre le terme plus ou moins compréhensible.

Les expressions métaphoriques abondent également dans la langue de la banlieue. Il s'agit donc d'insérer dans un contexte déterminé un élément concret dans un contexte abstrait par substitution analogique. Selon Lakoff et Johnson, les métaphores sont essentiellement culturelles et dans une grande mesure, propres à chaque langue: dans presque tous les cas, les métaphores sont l'expression de réalités abstraites en termes d'autres plus abstraites encore de l'univers d'action et des expériences humaines (1998). Nous reviendrons plus loin sur l'idée selon laquelle notre système conceptuel (en d'autres termes, notre vision du monde) est conformé par notre interaction constante avec notre environnement culturel et physique. Ainsi les jeunes de la banlieue n'hésitent point à recourir à la néologie lexicale pour donner naissance à des productions citées dans l'ouvrage Tchatche de banlieue, comme par exemple: 
«Cerveau fait demi-tour» faisant référence à une personne qui devient folle (1998:23). "Lavabo » qui désigne un alcoolique, c'est-à-dire une personne qui recherche, avale et ingurgite tout liquide alcoolisé (1998: 9).

La troncation, permettant de raccourcir un grand nombre de mots, constitue un autre procédé. Ainsi des termes plurisyllabiques deviennent monosyllabiques comme par exemple: biz venant de l'anglais «business» désignant dans le contexte de la banlieue, le trafic ou les affaires illicites.

Nous pouvons mentionner également l'aspect prosodique du langage des cités. S'agissant de faits qui échappent à l'articulation segmentale tels que l'intonation, l'accentuation, le rythme, le débit, les pauses, ils revêtent une grande importance dans la description de la langue de la banlieue. Nous savons tous que l'intonation joue un rôle important en français et qu'elle adopte des aspects fort différents en fonction des registres. Le langage dans un registre soutenu emploie une intonation relativement monotone; en revanche, le registre familier dans lequel s'insère la langue des cités connaît de nombreux changements de tons. Quant au débit et au rythme, en dépit du fait qu'ils ne constituent pas des phénomènes purement linguistiques, leur fonction est pour nous fondamentale. Or, il s'avère que la langue de la banlieue a également développé un autre rythme du français, lequel se manifeste surtout dans la prononciation. Nous pouvons parler dans ce cas d'une articulation très ouverte et très à l'arrière des voyelles, tel que l'affirme Louis-Jean Calvet (1994:52). Par ailleurs, l'accent de mot ou de phrase se fait sur l'avant-dernière syllabe. Le débit, lui, est rapide et entraîne donc des phénomènes de relâchement dans l'expression.

$\mathrm{Au}$ terme de ces considérations sur quelques aspects de la structure et du fonctionnement du parler de la banlieue, qui sont loin, nous en sommes consciente, d'avoir abordé toute la complexité de la question, nous allons dès à présent envisager la question identitaire. Nous l'avons vu, ce parler est en constante évolution et nous pourrions certainement affirmer que l'une de ses fonctions principales demeure la fonction cryptique ou peut-être même ésotérique. Il s'agit bel et bien d'une langue que les jeunes de la banlieue veulent secrète, qui ne doit pas être comprise au-delà de la cité. D'où, l'immense capacité de changement au fur et à mesure que certaines expressions deviennent «transparentes »; ainsi de nouvelles créations lexicales apparaissent pour remplacer celles qui ont été révélées. Les recours linguistiques employés supposent donc une attitude active de la part de la communauté linguistique et concernent particulièrement le lexique. Il n'en demeure pas moins que ces jeunes, scolarisés jusqu'à l'âge de seize ans, connaissent la norme. C'est alors qu'une question nous vient à l'esprit: pourquoi ne l'emploient-ils pas, ne la respectent-ils pas afin d'accéder au fameux statut de «locuteur standard» tout au moins? Cette interrogation nous renvoie directement au problème de l'unité sociale versus le conflit social. Une norme linguistique constitue par excellence le trait unificateur d'un groupe: tout individu est conscient de ce fait et décide donc de son propre gré d'appartenir à un groupe de locuteurs ou à un autre. Cela dit, même si les variantes du registre dit soutenu sont connues de tous, certains ne sont pas disposés à les adopter. Dans le cadre des populations de la banlieue, leur milieu social, leurs conditions de vie les amènent à se voir constamment en situation d'échec; l'élévation dans l'échelle sociale s'avère quasi impossible pour eux. C'est pourquoi, les variantes appartenant au registre soutenu et normatif sont associées au snobisme et sont considérées comme une forme d'hostilité à l'égard de leur famille, leurs amis et leur voisinage. Un grand 
nombre d'études ont montré que les réactions subjectives face aux variantes linguistiques sont différenciées par classe et vont bien au-delà d'une simple échelle de prestige. Citons à ce titre les études menées par Labov dans les quartiers de New York (1972). La langue académique est associée au groupe de locuteurs des classes sociales dominantes; la langue normative est utile pour l'obtention d'un emploi à responsabilité par exemple et permet d'accéder à la mobilité sociale. Nous sommes tous conscients de ces aspects puisqu'ils nous ont été inculqués au cours de notre scolarité et forment le discours quotidien des moyens de communication. Par contre, pour les jeunes de la banlieue, leur langue et par conséquent l'ensemble de ses variantes, possède un autre type de prestige: celui de la solidarité. Un individu utilisant leur parler appartient nécessairement à leur communauté.

Nous avons évoqué plus haut l'emploi fréquent de la métaphore. La langue de la banlieue exprime en effet la pensée, l'esprit d'un groupe de locuteurs, sa façon d'être et sa vision du monde. Nous rejoignons à ce titre, les propos de George Lakoff et de Mark Johnson (1998:39) selon lesquels la métaphore imprègne la vie quotidienne, non seulement le langage mais aussi la pensée et l'action. Notre système conceptuel ordinaire, selon lequel nous pensons et agissons, est fondamentalement métaphorique. Ainsi le système conceptuel joue un rôle essentiel dans la définition de la réalité quotidienne au sein des communautés de la banlieue. Cette dernière, étant envisagée comme sous-culture de la culture française, le choix des valeurs auxquelles on accorde la priorité relève donc de la sous-culture à laquelle on appartient et partiellement aussi des valeurs personnelles. Les métaphores employées dans ce parler ne font donc que révéler la vision du monde de la banlieue, ses valeurs, sa pensée.

Pour conclure ces quelques considérations, nous soulignerons qu'en dépit des multiples condamnations dont a été victime et continue de l'être le parler de la banlieue, à la fois cible des puristes de la langue française et source de leurs véhémentes imprécations, ce phénomène linguistique tend de plus en plus à dépasser ses sphères d'origine et s'érige en prédilection des moyens de communication, avides d'expressions proprement «banlieusardes».

Enfin pour terminer, nous citerons les propos d'Henriette Walter prononcés lors d'un entretien accordé aux auteurs de Tchatche de banlieue:

Pour les linguistes, lorsqu'il y a du nouveau dans une langue, il est prudent d'attendre quelques années avant de voir s'il s'agit d'une mode passagère ou si le phénomène va se fixer. Une précaution d'autant plus grande aujourd'hui avec la médiatisation qui accélère la circulation des idées. Lorsque les mots de la banlieue connaîtront la même carrière et entreront dans les dictionnaires, ils seront pour les futurs linguistes les témoins vivants de l'Histoire de notre pays en cette fin de siècle (1998: 127).

\section{RÉFÉRENCES}

BAYlon, C. (1996): Société, langue et discours, Paris, Nathan Université.

Blanche-Benveniste, C. (1997): Approches de la langue parlée en français, Paris, Collection

l'Essentiel Français, Ophrys.

Bourdieu, P. (1982): Ce que parler veut dire, Paris, Fayard.

Boyer, H. (1997): «Nouveau français, parler jeune ou langue des cités?», Langue française, $\mathrm{n}^{\circ} 114$, p. $22-26$.

CAlvet, L. J. (1994): Les voix de la ville. Introduction à la sociolinguistique urbaine, Paris, Plon. Ferguson, C. (1971) : Language, Structure and Language Use, Stanford, Stanford University Press. 
Labov, W. (1972): Language in the Inner City. Studies in the Black English Vernacular, Philadelphia, University of Pennsylvania Press.

Lakoff, G. and M. Johnson (1998): Metáforas de la vida cotidiana, Traducción de Carmen González Marín, Madrid, Catédra.

Merle, P. (1999): Le prêt à parler, Paris, Plon.

Muller, B. (1985): Le français d'aujourd'hui, Paris, Éditions Klincksieck.

Pierre-Adolphe, P. et J.-L. Bocquet (1997): Rapologie, Paris, Éditions Mille et une nuits.

Pierre-Adolphe, P., M. Mamoud et G. O. Tzanos (1998): Tchatche de banlieue, Paris, Éditions Mille et une nuits.

Vaillant, E. (1996) : L'immigration, Toulouse, Éditions Milan.

Vieillard-Baron, H. (1996): Les banlieues, Paris, Flammarion. 